[6a] Cf. Marshall's "Microbiology "(London, 1919), p. 417.

[7] Z. f. Immunitatsforsch. $u$. exp. Therap., 29, 343-8 (1920).

[8] Spertich. Centr. Bakt. Parasitenk. II, 34, 405-30 (1912).

[9] Jour. Microbiol. Petrograd, 3, 77-8, 273-90 (1916).

[10] Holm and Sherman. J. Bact., 7, 465-70, 583-8 (1922); Proc. Soc. Exp. Bio!. and Med., 21, 311 (1924).

[11] Abs. Bact., 3, 5, (1919).

[12] Michigan Agr. Stat. Rept. (1915), p. 209.

[12a] Clayton. Margarine (London, 1920, p. 127.

Fischer et Gruenert, Zeits. Nahr. Genussm., 22, 553 (1911).

[13] Storrs Agric. Exp. Stat. Bull. 79 (1914), 387-94.

[14] Bokorny. Centr. Bakt. Parasitenk, II abt., 35, 118.97.

Petersen. Arch. Hyg. 37, 171 (1900).

[15] J. Dairy Sci., 1, 114-26 (1917).

[16] Centr. Bakt., II Abt., 22, 32 (1909).

[17] U. S. Bur. Anim. Ind. Bull, 84 (1906), p. 17.

[18] The Butter Industry, p. 340.

[19] Centr. Bakt. II Abt., 22, 2232 (1909).

[20] Mohler, Washburn et Rogers. U.S.Dept. Agric., Bur. Anim. Ind., Annual Rept. (1909), pp. 179-85.

Hewlets. Manual of Bacteriology (London, 1921) pp. 409 et 749 ,

[21] U. S. Dept. Agric., Bur. Anim. Ind., Cire. 153 p. 38.

[22] The Butter Industry, p. 343.

[23] Science, 42, 319 (1915).

[23a] Mykologie der Milch, (1911), p, 210.

[24] The Butter Industry, p. 334.

[25] J. Dairy Sci., 3, 77-106 (1920).

[26] The Butter Industry, p. 519.

[27] J. Dairy Sci., 3, 103 (1920).

[28] Trans. Farad. Soe., 16, Appendix 22-6 (1921).

\title{
LE VERT JANUS EN FACE DU BLEU DE MÉTHYLÈNE DANS L'ESSAI A LA RÉDUCTASE SELON BARTHEL
}

\author{
par Léo SOEP
}

Laboratoire du Service de contrôle des Denrées alimentaires à Amsterdam

S'il était possible de mesurer l'importance d'un sujet par le nombre de pages qu'on a bien voulu y consacrer dans les livres et les périodiques, l'épreuve de la réductase occuperait, sans doute, une des premières places. Rien qu'une Bibliographie complète comprendrait plusieurs centaines de rappels de travạux. Tout bien considéré, il faudrait commencer en 1844 avec une notice de Heдmнодтz, qui observe que les bactéries de la putréfaction sont capables de réduire le tournesol (Loc. cit. CHRISTIANSEN, voir plus bas). Ainsi que des recherches récentes l'ont démontré (BARTHEL, entre autres) l'action réductrice que le lait exerce sur les matières colorantes, spécialement sur le bleu de méthylène, doit être spécialement attribuée à des influences bactériennes. Il est bien vrai 
que le lait produit dans des eonditions aseptiques (lait exempt de bactéries) montre un léger pouvoir réducteur, mais l'intensité du phénomène est insignifiante en comparaison avec celle de la réduction microbienne (1).

Bien qu'il ne soit pas nécessaire de le répéter que le bleu de méthylène ne constitue pas la seule matière colorante qui puisse être réduite par les bactéries du lait, il est pourtant utile de fixer l'attention sur ce point : que l'épreuve de la réductase et le bleu de méthylène ne sont pas en quelque sorte des frères siamois, mais que le pouvoir de réduction constitue une propriété très générale que nombre de bactéries possèdent, qui peut porter sur bien des matières colorantes et que, pour cette raison, la réduction du vert Janus n'est pas extraordinaire.

Il est remarquable de constater que la méthode originelle de BARTHEL pour faire l'épreuve de la réductase, s'est maintenue pendant environ 10 années, presque sans subir de modifications et quand on les notait, elles étaient légères et portaient notamment sur les quantités relatives de réactif. Le bleu de méthylène a un grand désavantage : le leuco-dérivé qui se forme à la suite de la réaction n'est pas stable et il subit la réoxydation au contact de l'oxygène de l'air qui diffuse à travers la surface des liquides, La période de réduction se trouve ainsi prolongée du fait de la réoxydation et cela est d'autant plus marqué que cette période est plus longue (2).

C'est pour cette raison que CHRistiansen (3) a proposé de remplacer le bleu de méthylène par le vert janus (safrano-azo-diméthylaniline) laquelle substance colorante est vert bleuâtre en solution acide (vert clair en solution alcaline) et qui se réduit en une combinaison d'un rouge clair, en passant par le violet, pour donner ensuite une substance incolore.

La matière rouge qui est formée en premier lieu est stable envers l'oxygène de l'air, tandis que la substance incolore, qui est formée ensuite, ne l'est pas, mais cela n'a pas d'importance.

On appelle période de réduction le temps qui s'écoule entre le commencement de l'essai et le moment où la formation de la coloration rouge est terminée. A 10 ccmo de lait, on ajoute 1 ccme d'une solution de 1 partie de matière colorante dans 10.000 parties d'eau. Pour le restant, ia procédure est identique à celle que l'on suit avec le bleu de méthylène. Il est très facile d'observer le moment où la réduction est complète.

Il semble que la modification de Christransen ait été acceptée par de nombreuses autorités laitières en Allemagne et dans les grandes firmes, notamment ehez Bolle à Berlin.

En ce qui concerne le jugement que l'on peut porter sur le lait à l'aide des résultats que donne le vert janus, il apparait que CHRISTIANSEn suppose

(1) Arkir for Kemi, t. 9, No. 19, 1925.

(2) La montée de la crôme a également une certaine influence sur la diffusion.

(3) Milchwirtschaftliche Zeitung, Lubeck, No. 42, 1926.

Molkerei-Zeitung, Hitdecheim, No. 102, 1926. 
qu'on peut appliquer les mêmes règles que dans le cas du bleu de méthylène ; en tous cas, cet auteur passe cette question sous silence et il importe cependant de l'envisager, car il n'est pas certain qu'il y ait un parallélisme dans les deux jugements que l'on peut porter, selon que l'on emploie, comme matière colorante devant être réduite, le bleu de méthylène ou le vert janus; il y a à cela plusieurs raisons :

10 Le retard de la réduction par l'oxygène de l'air fait défaut avec le vert janus et pour cette raison la période de réduction devrait être inférieure dans le eas où l'on emploierait cette matière colorante.

$2^{\circ}$ Toutes les matières colorantes constituent plus ou moins des poisons pour les bactéries, même à des faibles concentrations; elles retardent l'action des bactéries par absorption et d'autres phénomènes analogues. Cette influence est difficile à mesurer, on ne peut pas en prévoir ni l'étendue; ni même la direction.

Dans le but de trouver la réponse à la question de savoir dans quelle mesure les résultats des épreuves au bleu de méthylène ou au vert janus correspondent, j'ai fait environ 1000 essais de chacune de ces matières colorantes, les uns étant placés à côté des autres et j'ai comparé les résultats par la voie de la statistique.

Le tableau I les reproduit. J'ai renoncé à donner les chiffres in-extenso parce qu'ils occuperaient une dizaine de pages au moins, ce qui serait inutile. Le tableau donne une impression générale parfaitement suffisante pour cette courte étude.

I. Colonnes verticales.

\begin{tabular}{|c|c|c|c|c|}
\hline$\xi$ & $\xi^{2}$ & $f_{c}$ & $f_{c} \cdot \xi$ & $f_{c} \cdot \xi^{2}$ \\
\hline$+1 / 2$ & $1 / 4$ & 179 & $+89,5$ & 44,75 \\
\hline+1 & 1 & 135 & +135 & 135 \\
\hline$+11 / 2$ & $2^{1} / 4$ & 55 & $+82,5$ & 123,75 \\
\hline+2 & 4 & 79 & +158 & 316 \\
\hline$+21 / 2$ & $6^{1 / 4}$ & 50 & +125 & 312,5 \\
\hline+3 & 9 & 55 & +165 & 495 \\
\hline$+31 / 2$ & $121 / 4$ & 36 & +126 & 441 \\
\hline+4 & 16 & 47 & +188 & 752 \\
\hline$+41 / 2$ & $20^{1} / 4$ & 13 & $+58,5$ & 263,25 \\
\hline+5 & 25 & 11 & +55 & 275 \\
\hline$+51 / 2$ & $30^{1} / 4$ & 10 & +55 & 302,5 \\
\hline
\end{tabular}

Les colonnes verticales donnent les résultats avec le bleu de méthylène, groupés par demi-heure ; de la même façon, les lignes horizontales comprennent les chiffres des épreuves au vert janus, groupés également par demi-heure. La table ne comprend que les épreuves dont les temps de décoloration étaient inférieurs à 6 heures ; les demi-heures complètes ont 
été comptées vers le côté inférieur; les demi-heures dépassant ont été ramenées au chiffre immédiatement inférieur. De 1036 essais couplés qui ont été faits, il fallut en éliminer 4 parce que l'épreuve au blè de méthylène a demandé plus de 6 heures, 35 parce que l'épreuve au vert janus a demandé plus de 6 heures, 52 parce que les résultats avee l'une et l'autre de ces matières colorantes étaient également supérieurs à 6 heures. En somme, il y a donc 91 de ces essais laissés de côté ; il en reste 945 dont le tableau fait mention.

TABLEAU I

\begin{tabular}{|c|c|c|c|c|c|c|c|c|c|c|c|c|c|c|c|}
\hline & & & 275 & 179 & 135 & 55 & 79 & 50 & 55 & 36 & 47 & 13 & 11 & 10 & 945 \\
\hline$+41 / 2$ & $51 / 2-6$ & & 1 & 2 & 1 & 2 & 3 & 3 & 11 & 7 & 14 & 4 & 2 & 3 & 53 \\
\hline+4 & $5-5 \frac{1}{2}$ & & & & & & & & 2 & 1 & 1 & & 3 & 1 & 8 \\
\hline$+31 / 2$ & $41 / 2-5$ & 2 & 4 & 2 & & & 4 & 2 & 7 & 13 & 10 & 4 & 4 & & 50 \\
\hline+3 & $4-41 / 2$ & & & & 2 & 2 & 6 & 7 & 7 & 8 & 2 & 4 & & 3 & 41 \\
\hline$+21 / 2$ & $31 / 2-4$ & & & 5 & 4 & 5 & 14 & 15 & 6 & 3 & 11 & & & 1 & 64 \\
\hline$+21 / 2$ & $3-31 / 2$ & & 1 & 13 & 35 & 17 & 15 & 4 & 16 & 3 & 5 & & 1 & $=$ & 110 \\
\hline$+11 / 2$ & $21 / 2^{-3}$ & & 6 & 9 & 23 & 16 & 8 & 11 & 1 & 1 & 1 & & & & 77 \\
\hline$+1 \frac{1}{2}$ & $2-21 / 2$ & & 13 & 35 & 20 & 6 & 18 & 7 & 3 & & 1 & & & & 103 \\
\hline$+1 / 2$ & $11 / 2-2$ & & 13 & 36 & 17 & 3 & 2 & & 1 & & & & & 1 & 73 \\
\hline+0 & $1-1 \frac{1}{2}$ & & 71 & 43 & 15 & 2 & 3 & 1 & 1 & & 2 & & 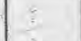 & & 138 \\
\hline$-1 / 2$ & $1 / 2^{-1}$ & & 83 & 25 & 8 & 1 & 4 & & & & & 1 & 1 & & 123 \\
\hline-1 & $0-1 / 2$ & & 83 & 9 & 10 & 1 & 2 & & & & & & I & & 105 \\
\hline déviat. & heures & : & $0-1 / 2$ & $1 / 2-1$ & $1-11 / 2$ & $11 / 2-2$ & $2-21 / 2$ & $21 / 2^{-3}$ & $3-31 / 2$ & $31 / 2^{-4}$ & $=4-41 / 2$ & $41 / 2-5$ & $5-51 / 2$ & $51 / 2-6$ & \\
\hline & dévial. & & 0 & $+1 / 2$ & +1 & $+11 / 2$ & +2 & $+21 / 2$ & +3 & $+31 / 2$ & +4 & +4 & +5 & $+51 / 2$ & \\
\hline
\end{tabular}

Essais du bleu de méthylène.

Si l'on peut supposer que le rapport soit linéaire, il s'en suit que le coefficient de corrélation, calculé selon la méthode de la régression linéaire, est 0,75. En effet, ce coefficient de corrélation est assez élevé, mais on pouvait s'y attendre, parce que les résultats ont la même orientation et qu'jls doivent croître ici dans le même sens.

Les finesses du calcul sont les suivantes : soit $f_{c}$ la valeur des chiffres dans les colonnes verticales, $\xi$ la déviation correspondante, $f_{l}$ la valeur 
des chiffres des lignes horizontales, $n$ la déviation correspondante, et soit enfin $n$ le nombre des couples considérés. Alors le calcul se compose de 3 parties distinctes, représentées dans les tables suivantes, lesquelles n'ont pas besoin d'explications ( 1 ).

Nous appelons colonne de référence celle montrant la déviation zéro, c'est donc la première. Ensuite nous appelons $d_{c}$ le quotient de la somme de tous les $f_{c}$. $\xi$ par $n$, donc : $\mathrm{d}_{c}=\frac{\Sigma f_{c} \xi}{n}=1,3095$, et d'une manière analogue nous désignons par $s_{c}$ lẹ quotient de la somme de tous les $f_{e} \xi^{2}$ par $n$, donc $\int_{c}=\frac{\Sigma f_{c} \cdot \xi^{2}}{n}=3,6622$.

Enfin nous dérivons $\sigma_{c}=\sqrt{{S_{c}^{2}-d_{c}^{2}}^{2}}=1,3955$.

\section{Lignes horizontales.}

Un calcul tout à fait analogue donne les valeurs correspondantes pour les lignes horizontales.

$d_{l}=\frac{\Sigma f_{l} \cdot \eta}{n}=1,0974 ; \quad \rho_{l}=\frac{\Sigma f_{l} \cdot \eta^{2}}{n}=3,6963 ; \quad \sigma l=\sqrt{s l^{2}-d l^{2}}=1,5786$

III. Produits. Fréquences (Les indices ont été omis maintenant):

$\begin{array}{ccccc}\begin{array}{c}\text { Produits } \\ \text { des déviations }\end{array} & \text { Positif } & \text { Négatif } & \text { Positif } & \text { Négatif } \\ \xi . n & f & f & f . \xi, n & f . \xi . n \\ 1 / 4 & 36 & 25 & 9 & 61 / 4 \\ 1 / 2 & 52 & 17 & 26 & 81 / 2 \\ 3 / 4 & 12 & 1 & 9 & 3 / 4 \\ 1 & 35 & 14 & 35 & 14 \\ 11 / 4 & 5 & - & 61 / 4 & - \\ 11 / 2 & 30 & 1 & 45 & 11 / 2 \\ 13 / 4 & 2 & - & 31 / 2 & -\end{array}$

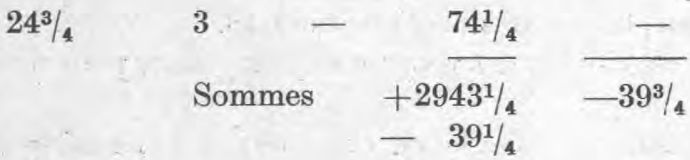

$$
\begin{aligned}
& \text { Alors: } \Sigma f . \xi \cdot n=+29131 / 2 \\
& \frac{\Sigma f \cdot \xi \cdot n}{n}=\frac{+2913 \frac{1}{2}}{945}=3,0831 \text {. }
\end{aligned}
$$

(1) C'est-à-dire en ce qui concerne le calcul. Je ne peux pas m'occuper d'explieations théoriques. Les lecteurs désireux de savoir plus sur ce eôté peuvent consulter: G. Udny Yule, An introduction to the theory of statistics. London 1911.; Truman L. Kelly, Statistical method, New-York 1923; E. Czuber, Dio Statistischen Forschungsmethoden; enfin pour les plus avancés: A. A. Tschuprow. Grundbegriffe und Grundprobleme der Korrelations Theorie, Leipzig 1925. 
Le coefficient de corrélation $C$ se calcule ensuite au moyen de la formule (BRAVAIS, PEARSON) :

$$
C=\frac{\frac{\Sigma f \cdot \xi \cdot n}{n}-d_{c .} d_{l}}{\sigma_{c \cdot} \sigma l}=\frac{3,0831-1,3095 . \quad 1,0974}{1,3955.1,5786}=0,75
$$

Nous pouvons encore calculer la variation de $C$ par la formule :

$$
\nu=\frac{1-e^{e}}{\sqrt{n}}=0,014 .
$$

Donc enfin : $C=0,75 \pm 0,01(4)$.

Ainsi que le tableau I le montre, la régression n'est en réalité nullement linéaire, mais il n'est pas nécessaire de tenir compte de cela. S'il faut maintenant attribuer la même valeur réelle aux résultats des deux modifications de l'épreuve de la réductase, nous pouvons exprimer cela mathématiquement en posant que leur proportion $=1$. Dans tous les autres cas, cette proportion est différente de l'unité. Le fait que les deux résultats sont très souvent en même temps ou plus élevés ou plus bas, conduit immédiatement à considérer que leur proportion $=1$. En calculant la proportion pour l'ensemble des 945 couples d'essais et en prenant la moyenne, nous obtenons la proportion la plus vraisemblable. Il est cependant inutile de faire tout ce travail, puisqu'il est possible de trouver une bonne approximation, laquelle suffit en pratique, en suivant une méthode plus facile de la façon suivante.

Nous choisirons un rectangle élémentaire quelconque de la table de corrélation (tableau I). Ainsi la colonne verticale $2-21 / 2$ et la ligne horizontale $2-21 / 2$ et nous prenons les moyennes qui, dans les deux cas, sont $9 / 4=21 / 4$. La proportion de ces deux moyennes est alors de 9/9 et elle se rencontre 18 fois dans la colonne verticale. Si nous faisons ensuite de tels calculs simples pour tous les rectangles de la même colonne et et que nous prenons la moyenne nous obtenons un chiffre que nous pouvons considérer comme représentant la proportion moyenne pour la colonne en question; dans le cas considéré elle est 1,18.

Le tableau II donne les valeurs des proportions moyennes pour toutes les colonnes verticales.

La proportion moyenne se ealcule en considérant l'ensemble des essais et on trouve $722,6: 945=0,76$, les résultats des épreuves au bleu de méthylène occupant le numérateur.

Le tableau II montre que la porportion des valeurs des deux épreuves n'est pas une constante, qu'à de petites valeurs avec le vert janus correspondent des résultats plus de deux fois plus élevés que celles qu'on obtient avec le bleu de méthylène, mais qu'avec des valeurs plus élevées, les résultats se rapprochent de plus en plus et que les résultats avec le bleu de méthylène deviennent même supérieurs à ceux du vert janus. L'influence de la diffusion de l'oxygène de l'air y est donc bien évidente. 
Si la réduction a lieu en moins d'une demi-heure, on peut laisser tranquillement hors de considération l'influence de l'oxygène de l'air, parce que les propriétés spécifiques des bactéries décolorantes sont ici prépondérantes. Nous pouvons donc supposer comme vrai que le bleu de méthylène est réduit en moyenne plus de deux fois plus vite que le vert janus.

TABLEAU II

$\begin{array}{rccc}\text { Colonne } & \text { Somme des produits } & \text { Nombre } & \text { Proportion moyenne } \\ 0-1 / 2 & 129,04 & 275 & 0,469 \\ 1 / 2-1 & 113,37 & 179 & 0,627 \\ 1-1 \frac{1}{2} & 127,63 & 135 & 0,945 \\ 11 / 2-2 & 41,98 & 55 & 0,763 \\ 21 / 2-21 / 2 & 87,54 & 79 & 1,181 \\ 21 / 2-3 & 41,48 & 50 & 0,830 \\ 31 / 2-31 / 2 & 48,77 & 55 & 0,887 \\ 31 / 2-4 & 30,41 & 36 & 0,845 \\ 4-41 / 2 & 51,36 & 47 & 1,093 \\ 41 / 2-5 & 18,10 & 13 & 1,392 \\ 5-51 / 2 & 17,87 & 11 & 1,625 \\ 51 / 2-6 & 15,05 & 10 & 1,505\end{array}$

Il s'en suit, en outre, que les résultats des deux épreuves n'ont pas la même signification et qu'il n'est même pas possible de passer des uns aux autres d'une façon simple. En particulier, il n'est pas permis d'appliquer le classement des laits suivant le procédé de BARTHEL-ORLA JENSEN aux épreuves au vert janus, car on serait conduit à des résultats incorrects ce qui est très bien illustré par le Tableau III, dont il est inutile de donner l'explication, les chiffres parlant d'eux-mêmes.

TABLEAU III (total des 1.036 essais couplés)

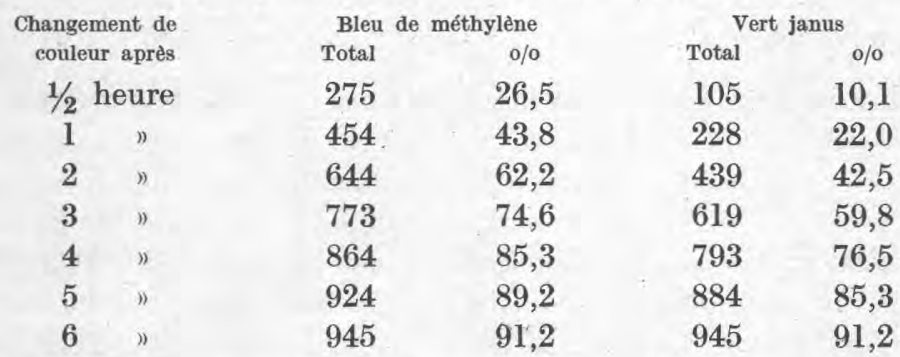

Nous rappelleronsici que le classement suivant BARTHEL-ORLA JENSEN, tel qu'il est appliqué dans les pays scandinaves pour le jugement-des laits, dans le sens technique aussi bien que dans le sens économique, distingue :

Classe 1 : Bon lait: réduction dans 5 h. $1 / 2$ au minimum; nombre de germes inférieurs à $1 / 2$ de million. 
Classe 2 ; Lait de qualité moyenne : 2 heures à $51 / 2$ heures; $1 / 2$ à 4 millions de germes.

Classe 3 : Mauvais lait : $1 / 3$ heure à 2 heures; 4 à 20 millions de germes.

Classe 4 : Très mauvais lait : moins de 20 minutes, plus de 20 millions de germes.

Christransen fait la distinction d'une façon légèrement différente et admet des temps de décoloration de 0 à 1 heure, 1 heure à 3,3 à 6 heures et plus de 6 heures. Dès l'instant qu'il a employé la méthode au vert janus, il est évident qu'il doit " sentir » le temps le plus grand de la réduction dans le cas du vert janus, bien que, cependant, il ne touche pas à cette question en particulier.

Remarques $1^{0}$ Tout d'abord, j'avais l'intention d'étendre cette petite étude sur beaucoup plus d'échantillons et de spécifier l'examen statistique, en tenant compte de l'origine du lait (producteur, laiterie, détaillant) mais des cireonstances imprévues ont rendu ce projet impossible. Les chiffres dont je pouvais disposer au moment de l'interruption du travail ne me permettaient pas une analyse aussi détaillée et je n'y mentionnais que les différences ;

$2^{\circ}$ Les essais ont été exécutés pendant les mois d'août-septembre 1927 ; la température a été très anormale, très inférieure à la température d'un été normal et un grand nombre d'essais qui ont donné un résultat faible, ne s'expliquent donc pas par des circonstances atmosphériques.

$3^{\circ}$ Les échantillons examinés étaient des échantillons normaux comme on les apporte régulièrement au Laboratoire du Service du Contrôle des denrées alimentaires à Amsterdam, sans aucun tamisage. Les chiffres donnés se rapportent au lait comme il vient au marché d'Amsterdam.

$4^{\circ}$ Des considérations comme les précédentes démontrent la grande utilité de recherches statistiques eomparatives.

Résumé : I. L'épreuve de la réductase suivant BaRTHEL donne des résultats trop élevés et d'autant plus que le chiffre correspondant est plus fort. Ce phénomène s'explique par la diffusion dans le liquide examiné de l'oxygène de l'air et par la réoxydation correspondante de la leucocombinaison formée par la réduction du bleu de méthylène. Pour cette raison, il est préférable de substituer une autre matière colorante au bleu de mythélène. Christiansen a proposé l'emploi du vert janus, matière colorante vert bleuâtre en solution acide et qui donne lieu à la formation d'une substance rouge par réduction.

II. Des essais comparatifs ont justifié l'emploi du vert janus.

III. Les résultats des deux modifications de l'épreuve de la réductasen'ont pas la même signification et l'échelle de BARTHEL-ORLA JENSEN n'est pas applicable aux essais au vert janus. 
IV. Christiansen distingue 4 phases:

0 à 1 heure - très mauvais

1 à 3 heures - mauvais

3 à 6 heures - qualité moyenne

plus de 6 heures - bon

Ainsi que le calcul statistique l'a démontré, ce classement correspond exactement à celui de BARTHEL-ORLA JENSEN pour l'épreuve au bleu de méthylène.

\title{
LES ENZYMES DU LAIT ET LEUR UTILISATION POUR APPRÉCIER LE CHAUFFAGE DU LAIT
}

\author{
par C. LIND. \\ Bactériologiste de laiterie, à Copenhague (Danemark)
}

Dans le contrôle pratique du lait, on a souvent besoin de déterminer si le lait a été chauffé ou non. C'est souvent là une chose difficile, parce que l'influence de la chaleur sur le lait varie avec la température et, d'autre part, le lait à examiner peut être un mélange de lait chauffé et de lait cru. Les questions auxquelles on doit répondre sont les suivantes :

$1^{\circ}$ Le lait a-t-il été chauffé à moins de $80^{\circ} \mathrm{C}$., c'est-à-dire pasteurisé à " haute température " comme l'exige la loi danoise, quand il est vendu sous le qualificatif "pasteurisé "?

Le lait a-t-il été chauffé momentanément à des températures inférieures à $80^{\circ}$ ?

$3^{0}$ Le lait a-t-il été chauffé pendant un temps défini à $62-64^{\circ}$, c'està-dire a-t-il subi ce qu'on appelle la "pasteurisation basse "?

$4^{\circ}$ Le lait est-il un mélange de lait chauffé et de lait cru?

$5^{\circ}$ Le lait est-il complètement cru, c'est-à-dire n'a-t-il subi aucun chauffage, quel qu'il soit?

La détermination du chauffage à $80^{\circ}$ ou au-dessus n'est pas difficile, elle s'effectue avec la méthode de STORCH (paraphénylenèdiamine et eaux oxygénée).

Mais il n'en est plus de même avec la pasteurisation basse, qui est très répandue sauf au Danemark. Un lait ainsi pasteurisé est, comme on le sait, chauffé une demi-heure à $62-64^{\circ}$. Il a partiellement conservé les qualités chimiques et biologiques du lait cru, ce que les médecins et surtout les pédiâtres considèrent comme très important pour l'élevage de l'enfance. Avec la méthode de STоRCH, il est impossible de contrôler le chauffage du lait qui a subi la pasteurisation basse, ainsi qu'un chauff age momentané à des températures inférieures à $78^{\circ}$, ou des mélanges de lait cru avec du lait ehauffé. Avee la méthode de STorcH, un lait chauffé momentanément à des températures au-dessous de $78^{\circ}$ et un lait provenant du mélange de lait pasteurisé avec au moins $10 \%$ 